Brit. J. Ophthal. (1955), 39, 380.

\title{
RETINAL IMAGE IN AXIAL AND REFRACTIVE AMETROPIA*
}

\author{
BY \\ JOSEPH I. PASCAL \\ New York, N.Y.
}

THERE are some features relative to the size of the retinal images in these two conditions which are of general interest, though they are rarely discussed.

Axial Ametropia.-The "basic" (blurred) retinal image is smallest in hyperopia, intermediate in emmetropia, and largest in myopia. The size of a blurred retinal image is best taken from the points in the diffusion circles at the extremities of the image where the nodal ray strikes the retina. We call this the "basic" retinal image. The increase or decrease in size of the "basic" image over its size in emmetropia is about 2 per cent. for every dioptre of error. When the correcting lens is placed at $F_{1}$ of the eye, the resultant clear retinal image in hyperopia and myopia is the same size as that in emmetropia.

Refractive Ametropia.-The " basic" retinal image is similarly smaller in hyperopia and larger in myopia than that in emmetropia. The decrease or increase in size is much less than in the axial type, being about 0.5 per cent. for every dioptre of error. In a medium grade of refractive error, say $4 \mathrm{D}$, this will amount to about 2 per cent. This statement is contrary to the general teaching of most texts, but it can be proved by calculating the position of the nodal point in refractive ametropia.

If we use the reduced schematic eye and take $5.5 \mathrm{~mm}$. as the radius of the "reduced" refracting surface in emmetropia, we find that the radius for $3 \mathrm{D}$ of hyperopia is $5.79 \mathrm{~mm}$., and that for $3 \mathrm{D}$ of myopia is $5.24 \mathrm{~mm}$. The percentage magnification or reduction of the "basic" retinal image can be calculated from the displacement of the nodal point which is the centre of curvature of the reduced refracting surface.

There are three nodal points to be considered. Their distances from the reduced refracting surface are $5.24 \mathrm{~mm}$. (in $3 \mathrm{D}$ myopia), $5.5 \mathrm{~mm}$. (in emmetropia), and $5.79 \mathrm{~mm}$. (in $3 \mathrm{D}$ hyperopia). The corresponding distances form the retina are $16 \cdot 21,16 \cdot 50$, and $16 \cdot 76 \mathrm{~mm}$. The retinal images are in the ratio of these distances from the retina, viz. 16.21 :16.50: 16.76. This causes a change in the size of the basic retinal image as compared with that in emmetropia. It causes an increase of about 0.5 per cent. in myopia and a decrease of about 0.5 per cent. in hyperopia for every dioptre of error.

The correcting lens in axial ametropia changes the size of the "basic" retinal image. A plus lens enlarges it, a minus lens reduces it. But when the

*Received for publication December 28, 1954. 
lens is placed at $F_{1}$ of the eye, the final clear retinal image is the same size in hyperopia and myopia as in emmetropia.

But it is quite different in refractive ametropia. The plus correcting lens, whether at $F_{1}$ or not, produces a clear retinal image larger than that in emmetropia by about 1.7 per cent. for every $+1 \mathrm{D}$ of correction. The minus correcting lens produces a clear retinal image which is smaller than that in emmetropia by about 1.6 per cent. for every $-1 \mathrm{D}$ of correction. The resultant clear corrected retinal images are not all equal to each other as they are in axial ametropia.

In axial ametropia, magnification or reduction involves only one mode of comparison, the "basic" blurred retinal image in ametropia, with the clear corrected retinal image in emmetropia or corrected ametropia. The images here are all the same size. In refractive ametropia there are two modes of comparison. One involves the magnification or minification of the "basic" blurred retinal image in relation to the image in emmetropia. The other involves the magnification or minification of the clear corrected image in ametropia in relation to the image in emmetropia.

In a sense then the patient who has refractive hyperopia has an advantage over the patient with an equal amount of axial hyperopia. In the refractive type the plus correcting lens enlarges the retinal image relative to its size in emmetropia, by about 1.7 per cent. for every $+1 \mathrm{D}$ of correction. In the axial type the plus correcting lens produces a clear retinal image of the same size as in emmetropia. It is the reverse in myopia. The patient who has axial myopia has some advantage over the patient who has the same amount of refractive myopia. In the axial type the minus correcting lens produces a clear retinal image of the same size as in emmetropia. In the refractive type the minus correcting lens produces a smaller retinal image than in emmetropia, the reduction in size being about 1.6 per cent. for every $-1 \mathrm{D}$ of correction.

These differences have some bearings on clinical practice. They help to explain the different reactions of patients having the same degree of ametropia, also variations in the degree of corrected visual acuity, and so on.

\section{Summary}

In uncorrected axial ametropia, the "basic" retinal image is smaller in hyperopia and larger in myopia than that in emmetropia by about 2 per cent. for every dioptre of error. In corrected axial ametropia, the retinal image is the same size in all three conditions.

In refractive ametropia the "basic" retinal image is smaller in hyperopia and larger in myopia than that in emmetropia by about 0.5 per cent. for every dioptre of error. The correcting plus lens makes the retinal image larger in hyperopia and smaller in myopia than that in emmetropia by about 1.7 to 1.6 per cent. for every dioptre of correction. These features place the refractive hyperope and the axial myope in a more favoured condition for effective seeing. 